\title{
Lo storico e il suo biografo
}

\section{La vita di Eric Hobsbawm secondo Richard Evans}

Ingram: per dare ai lettori un'idea della biografia del famoso storico Eric J. Hobsbawm, scritta da Richard J. Evans si può partire da questo nome. Per molti italiani, è una marca di camicie. Ma per chi dovesse leggere „La spia perfetta“, romanzo di John Le Carré del 1986, è anche il nome di un personaggio minore, un funzionario dei servizi segreti inglesi, presente a una riunione con i colleghi della CIA: non dice una parola e non ricompare più in seguito. Talmente minore, che di lui nel romanzo c'è solo il nome.

„A perfect spy“ comparve nel maggio 1986 e a p. 233 quel personaggio si chiamava Hobsbawn (con la n). Avvisato da un amico, Eric Hobsbawm scrisse subito a Le Carré: per favore cambi quel nome nelle prossime edizioni, magari qualcuno prenderà la cosa letteralmente, è imbarazzante per un uomo di sinistra come me (ma posso chiederle come le è venuto in mente proprio quel nome? si tratta forse di un'allusione esoterica?).

Benché Evans dica il contrario, Hobsbawm fu accontentato da Le Carré, anche se non per tutte le traduzioni (troppo difficile): Ingram, dunque, al posto di Hobsbawn.

Il libro-monumento di Evans - Regius Professor a Cambridge, storico di mestiere, autore di libri fondamentali sulla Germania nazista - contiene moltissime storie come questa. In primo piano c'è il carattere di Hobsbawm, la persona in un momento qualsiasi della vita quotidiana: qui preoccupato per la sua reputazione di sinistra, per la piccola macchia di un oscuro personaggio quasi omonimo in una spy-story - altrove in una luce migliore: per esempio come ospite impeccabile, divertente, nelle cene a casa sua a Nassington Road, a Londra, conversatore brillantissimo, sorridente. A suo agio, dopo cena, nel mandare lui la lavastoviglie continuando a chiacchierare con gli amici (a volte capi di governo, o premi Nobel).

Nato nel 1917, morto nel 2012, Hobsbawm ha avuto una vita lunghissima, e ha pensato anche al problema del suo archivio, delle sue carte private e della loro utilizzazione. Con i suoi collaboratori, aveva previsto prima una biografia autorizzata - da pubblicare dopo la morte della seconda moglie Marlene - poi studi specifici indipendenti. Chiamato a commemorare accademicamente il collega scomparso, Evans ha poi pro-

Recensione di: Richard J. Evan s, Eric Hobsbawm. A Life in History, London (Little, Brown Book Group) 2019, XIII, 785 pp., ill., ISBN 978-1-4087-0741-8, GBP 30.

Kontakt: Massimo Mastrogregori, mastrogregori@yahoo.com 
seguito l'opera nel quadro di una biografia autorizzata, subito pubblicata con il consenso della vedova. Ora seguiranno gli studi indipendenti, che sono in corso.

C'era però un problema: che c'è di interessante in una vita di studio, in cui per lo più si legge, si scrive, si fa lezione, si viaggia per ricerche e convegni? Si legge, soprattutto: attentamente, voracemente. Ecco un'altra istantanea: sull'autobus che lo riporta a casa dall'università, Hobsbawm è assorto, a capofitto in un libro. A una fermata, sale la figlia, che vorrebbe salutarlo, ma lui prosegue a leggere indisturbato, nemmeno se ne accorge.

L'autobiografia scritta da Hobsbawm stesso offriva una soluzione: interessanti sono i tempi, in cui lo storico è vissuto („Interesting times“, New York 2002). In essa pochissimo è riferito di ciò che gli è davvero accaduto. Come è stato osservato, il libro riscrive quello sul secolo breve da un punto di vista un po’ più personale („Age of extremes“, London 1994).

Evans risolve il problema brillantemente, in modo radicalmente opposto. Sulla scena è la persona, quello che gli succede, quello che fa. Certo, ci sono nel libro gli elementi di una biografia intellettuale - la presentazione cronologica delle opere, la descrizione del loro contenuto, che cosa ne hanno detto i critici. Ma questa è la parte di routine.

Il suo vero scopo è quello di una biografia vera e propria: mostrare quanto è interessante la vita di una persona per sé stessa - non come esempio della vita di uno storico, non come strumento di conoscenza del Novecento.

Biografia vera e propria, cioè non strumentale. Biografia come la pensava Virginia Woolf, un racconto che ci dica anche come si vestiva quella persona, in che case abitava, chi erano le zie - soprattutto le zie („The art of biography“ 1938 [London 1942]).

Dalla presentazione concreta, visiva, della persona e delle sue vicende private il lettore potrà poi muovere per infinite strade verso altri problemi, altri interrogativi. Ma è qui, nella presentazione della vita quotidiana il cuore del lavoro di Evans.

Il biografo ha avuto accesso a fonti private, diari in tedesco, lettere familiari, racconti, poesie e confessioni, e anche resoconti finanziari, e ha restituito la dimensione privata esplicitamente, senza arretrare neanche di fronte a particolari intimi, come quelli sessuali.

La presentazione è più rapida per i primi anni, quando Hobsbawm visse ad Alessandria d'Egitto, Vienna e Berlino. Gli anni del liceo a Londra e dell'università a Cambridge, tra i sedici e i ventidue, sono quelli descritti più densamente, nei capitoli $2 \mathrm{e}$ 3 (studente di successo). Un maggior numero di note documenta gli anni dai trenta ai quarantacinque, l'inizio della carriera di storico, nei capitoli 5 e 6 (avvio di carriera difficile).

Pochissimo uso è fatto degli scritti su Hobsbawm: non apprenderemo da Evans che la sua è la terza biografia consacrata al personaggio. Il lavoro è svolto prevalentemente sulle carte dell'archivio privato. Un notevole spazio è anche dato alle notizie 
provenienti dal fascicolo Hobsbawm del servizio segreto inglese (in parte ancora inaccessibile).

Come persona, Hobsbawm manifesta prestissimo un'attrazione irresistibile per la lettura e una tendenza al dialogo interiore e alla riflessione, anche scritte (il diario in tedesco, appunto: la sua lingua-rifugio). Il mondo crolla intorno al giovane ragazzo ebreo che non ama la propria figura fisica. Uno dopo l'altro muoiono i genitori, poi deve lasciare Berlino, ambiente troppo ostile, dalla primavera del 1933. L'adesione al movimento comunista avviene a Berlino e matura poi a Londra: la fedeltà a questa nuova comunità - globale, larghissima, internazionale, in parte reale, in parte immaginata - non si interromperà mai. Come ha osservato Donald Sassoon, essa è un dato psicologico centrale della sua identità. Il fatto di essere comunista in Inghilterra lo ha ostacolato, prima nel servizio militare durante la guerra, poi nella carriera di studioso. Aver saputo resistere a queste pressioni ha rinforzato tale identificazione personale in un movimento collettivo.

Leggere e scrivere sono la sua vita, e Hobsbawm ci riesce molto bene fin dagli anni della scuola e dell'università: ha uno stile vivace, attraente, pungente. Lo si vedrà anche nelle grandi sintesi sulla storia e civiltà del capitalismo, pubblicate tra il 1961 e il 1994: lavori costruiti, per ammissione stessa dell'autore, su materiali di seconda o terza mano, eppure quanto mai originali e „orientati“. L'idea di avviarsi alla carriera degli studi storici arriva piuttosto tardi.

A prima vista c'è qualcosa di paradossale, forse di indebito, nel restringere in una dimensione privata, quotidiana, esistenziale, la figura di un intellettuale comunista e marxista come Hobsbawm. Le vicende biografiche personali - per esempio la sua infelicità con la prima moglie, le scorribande notturne nel mondo del jazz londinese a Soho, poi la vita familiare più stabile con Marlene e i figli - non sono irrilevanti per lo studio dell'organizzazione della cultura, delle sue condizioni, delle sue strutture? Non bisognerebbe prendere atto che il lavoro di Hobsbawm fu prima di tutto politico e lasciare da parte i particolari?

Su questo punto il libro di Evans presenta, forse inevitabilmente, qualche oscillazione, qualche asimmetria. Non si può chiedere al biografo Evans di essere altrettanto marxista del personaggio Eric. E i tempi sono molto cambiati. Autori e pubblico fanno ora i conti con la scomparsa dei destini collettivi e con l'asfissiante molteplicità di soggettività disperse, isolate, petulanti (comprese quelle degli storici).

Nel libro di Evans, quindi, c’è Eric Hobsbawm il politico, il comunista globale, almeno quanto la persona privata. I due piani però non si avvicinano facilmente.

Il comunista globale non fa che pensare ai cambiamenti veri, alle rivoluzioni. Da ragazzo scrive un piano dettagliato della rivoluzione comunista nella zona di Londra. In seguito allarga lo sguardo, dalla metropoli al mondo intero, con strumenti sempre più raffinati e scientifici, ma continua a fare essenzialmente la stessa cosa. Non è interessato alle riforme (a sorpresa, nel saggio sui „Primitive Rebels“ del 1959, chiama riformista addirittura la mafia). 
Con occhio non disinteressato, negli anni Cinquanta studia i banditi e ribelli „sociali“, quelli che raddrizzano i torti e donano ai poveri quello che rubano ai ricchi. Sotto i suoi occhi, nei due decenni successivi, tale fenomeno si arricchisce di una componente politico-militare organizzata, e diventa la guerriglia sudamericana, rurale, poi urbana, poi anche europea.

Come storico, si definisce un „guerrilla historian“: la matrice antagonista, politica e rivoluzionaria del suo lavoro è piuttosto chiara, anche se nel corso degli anni Settanta diventa un critico della guerriglia come mezzo per impadronirsi del potere e loda piuttosto i fronti popolari, e particolarmente il „compromesso storico“ proposto dai comunisti italiani (considera il Pci „la grande storia di successo del movimento comunista mondiale“ e con esso collabora in attività di propaganda).

Il suo rapporto con il movimento comunista mondiale sembra quello di un outsider, abbastanza fuori linea rispetto al partito comunista inglese, più vicino alle concezioni del partito italiano, al quale non era iscritto. Ma ci si può domandare quando sia effettivamente finito il lavoro di propaganda che svolgeva dalla fine degli anni Trenta per la macchina propagandistica progettata e avviata da Willi Münzenberg - lavoro al quale lo avevano introdotto, da ragazzo, Margot Heinemann e James Klugmann. E anche quanto dei suoi interessi e dei suoi viaggi si trovi a coincidere con i centri di interesse del movimento comunista internazionale (quello sovietico in particolare).

Rispetto al comunista globale, la persona privata ha un itinerario più tradizionale: nei primi anni Sessanta mette su famiglia, lavora, si arricchisce, compra una bella casa, piano piano si adatta, diventando un membro influente dell'establishment inglese (che c’è di più conservatore dell'establishment inglese?).

Non credo che Evans abbia mai pensato a una conciliazione di questi due piani diversi - di questi due mondi diversi. Ma resta il fatto che egli costruisce molto attentamente, nei primi capitoli, una figura di Hobsbawm come intellettuale rivoluzionario bolscevico, che dopo il 1956 perdiamo un po' troppo di vista, fino alla luce che semplicemente si spegne, per il nostro personaggio, col crollo del Muro, con le sue incalcolabili conseguenze negative (dissolvimento della tradizione illuminista, religione che riconquista una „presa“ sulle persone, dominio incontrastato del capitalismo come forza di distruzione).

Evans ha compiuto una ricerca magistrale sui materiali disponibili e ha scritto un libro molto bello. Il „guerrilla historian“ che ci presenta è un personaggio unico, che scrive per tutti, tranne i suoi colleghi, impegnati, secondo il buon metodo, soprattutto a scoprire e a interpretare nuove fonti negli archivi. Come professore, non ebbe quasi veri allievi.

A differenza della stragrande maggioranza degli studiosi di storia, Hobsbawm aveva un pubblico vastissimo, globale, un agente letterario (lo stesso di John Le Carré), un editore personale devoto, editori che gli pagavano anticipi con cinque zeri, e molti giornali e programmi televisivi a disposizione. Ha scritto libri interessanti, mentre il movimento comunista - la comunità che si era scelto - si divideva e poi scompariva. 
La sua opera è ora patrimonio comune, ma ancora soprattutto di una sinistra abbastanza dispersa. Con l'esempio ci ha ricordato che non è vero che la storia la scrivono i vincitori. I migliori storici sono gli sconfitti. 